\title{
Role of ORAl calcium release-activated calcium modulator 1 (ORAI1) on neutrophil extracellular trap formation in dairy cows with subclinical hypocalcemia
}

\author{
Bingbing Zhang, ${ }^{1 *} \odot$ Xinru Ma, ${ }^{2 *} \odot$ Juan J. Loor, ${ }^{3} \odot$ Qianming Jiang, ${ }^{3} \odot$ Han Guo, ${ }^{2} \odot$ Wei Zhang, ${ }^{1}$ Ming Li, ${ }^{2}$ \\ Xinquan Lv, ${ }^{1}$ Yufeng Yin, ${ }^{2}$ Jianan Wen, ${ }^{1}$ Jingjing Wang, ${ }^{1}$ Chuang $X u,{ }^{2} \dagger \odot$ and Wei Yang ${ }^{2} \odot$ \\ ${ }^{1}$ College of Life Science and Technology, Heilongjiang Bayi Agricultural University, Daqing 163319, China \\ ${ }^{2}$ College of Animal Science and Veterinary Medicine, Heilongjiang Bayi Agricultural University, Daqing 163319, China \\ ${ }^{3}$ Mammalian NutriPhysioGenomics, Department of Animal Sciences and Division of Nutritional Sciences, University of Illinois, Urbana 61801
}

\begin{abstract}
Hypocalcemia in dairy cows is associated with decreased neutrophil phagocytosis, adhesion capacity, migration, and reactive oxygen species (ROS) production through alterations in ORAI calcium release-activated calcium modulator 1 (ORAI1). Neutrophils can resist the invasion of pathogenic microorganisms by releasing neutrophil extracellular traps (NET). However, the mechanisms controlling NET formation during hypocalcemia are unknown. To address the role of ORAI1 in NET formation, neutrophils were isolated at $2 \mathrm{~d}$ postcalving from lactating Holstein dairy cows $(\mathrm{n}=$ 10 per group) diagnosed as clinically healthy (control) or with plasma concentrations of $\mathrm{Ca}^{2+}<2.0 \mathrm{mmol} / \mathrm{L}$ as a criterion for diagnosing subclinical hypocalcemia (SCH). A series of ex vivo experiments were conducted as follows: first, neutrophils isolated from both groups of cows were treated with phorbol 12-myristate 13-acetate (PMA) to stimulate NET formation; second, neutrophils from control and SCH were pretreated with or without the ROS scavenger $N$-acetylcysteine (NAC), the sarcoendoplasmic $\mathrm{Ca}^{2+}$ ATPase inhibitor thapsigargin, or ORAI1 blocker 2APB and then treated with PMA to stimulate NET formation; and third, neutrophils were transfected with small interfering (si)ORAI1 or nontarget siRNA (siNEG) and then stimulated with PMA to induce formation of NET. A one-way ANOVA was used for statistical analysis of individual experiments. In the first experiment, neutrophils from SCH cows formed NET with fewer DNA filaments, more diffused nuclei, and reduced translocation of myeloperoxidase (MPO) and neutrophil elastase (NE) to the nucleus. Neutro-
\end{abstract}

Received July 21, 2021.

Accepted December 15, 2021.

*These authors contributed equally to this work.

†Corresponding author: xuchuang7175@163.com phils from SCH cows stimulated with PMA had a lower mitochondrial permeability, the state of mitochondrial permeability transition pore was open, ROS production was lower and there was increased mitochondrial damage. In the second experiment, in both control and SCH-PMA stimulated neutrophils, exogenous NAC decreased NET formation (assessed via Hoechst 33342 dye; Beyotime). Furthermore, following the challenge with PMA, thapsigargin increased NET formation and ROS production, but blocking ORAI1 with 2APB decreased NADPH oxidase activation, ROS production, and NET formation. In the third experiment, neutrophils transfected with siORAI1 before stimulation with PMA had lower intracellular concentrations of $\mathrm{Ca}^{2+}$, NET formation, and ROS production. Overall, the data indicated that SCH reduces NET formation in neutrophils partly due to damaged mitochondria. The reduction in ORAI1 abundance in neutrophils of dairy cows with hypocalcemia also decreases ROS production.

Key words: dairy cattle, hypocalcemia, neutrophils, NET formation

\section{INTRODUCTION}

Neutrophils, the most-abundant innate immune effector cells of the immune system, neutralize and kill pathogens through the formation of neutrophil extracellular traps (NET) that form fiber-like structures composed of histones, protein granules, and doublestranded DNA (Wei et al., 2019). The NET structure contains DNA as the backbone to which granules of myeloperoxidase (MPO), cathepsin G, neutrophil elastase (NE), histone H3, and antimicrobial peptides attach (Ravindran et al., 2019). The formation of NET requires production of NADPH, which leads to synthesis of mitochondrial reactive oxygen species (ROS; Wang et al., 2019; Lood et al., 2016). In turn, ROS trigger activation and translocation of NE from azurophilic granules to the nucleus by stimulating MPO, leading 
to histone degradation and disruption of chromatin packaging (Papayannopoulos et al., 2010; Brinkmann, 2018).

High-producing dairy cows are most susceptible to subclinical hypocalcemia (SCH) during the transition from late pregnancy to lactation (Neves et al., 2018). Subclinical hypocalcemia is defined as blood $\mathrm{Ca}^{2+}$ of 1.38 to $2.0 \mathrm{mM}$ at 1 to $2 \mathrm{~d}$ postcalving without clinical symptoms, and its incidence can be as high as $30 \%$ to $45 \%$ within 2 wk postpartum (Thilsing-Hansen et al., 2002; Goff, 2008; Jawor et al., 2012). In addition to its close association with inflammation (Martinez et al., 2014), SCH decreased intracellular $\mathrm{Ca}^{2+}$ concentration and led to downregulation of ORAI calcium release-activated calcium modulator 1 (ORAI1) abundance via $1,25(\mathrm{OH})_{2} \mathrm{D}_{3}$-parathyroid hormone (PTH) action in dairy cows (Zhang et al., 2019). ORAI calcium releaseactivated calcium modulator 1 is a plasma membrane $\mathrm{Ca}^{2+}$ influx regulator protein that is associated with store-operated calcium entry, which opens upon binding to the stromal interaction molecule, a $\mathrm{Ca}^{2+}$ sensor located in the endoplasmic reticulum (ER; Immler et al., 2018). Our previous studies indicated that failure of neutrophils to uptake $\mathrm{Ca}^{2+}$ due to downregulation of ORAI1 during SCH is a factor contributing to impaired neutrophil function including migration, chemotaxis, adhesion, and phagocytosis (Zhang et al., 2019). The central role of intracellular $\mathrm{Ca}^{2+}$ on triggering a controlled inflammatory response in response to noxious stimuli has been documented in nonruminant cells (Chen et al., 2020).

Although it is known that the mRNA abundance of ORAI1 is decreased by $1,25(\mathrm{OH})_{2} \mathrm{D}_{3}$ and PTH and leads to reduced neutrophil activity including migration, adhering capacity, phagocytosis rate, and ROS production (Zhang et al., 2019), the role of ORAI1 in NET formation is unclear. The hypothesis for the present study was that SCH reduces ORAI1 in neutrophils and decreases ROS-dependent NET formation because of mitochondrial damage. To address this hypothesis, the specific objectives were to measure ORAI1 abundance, NET formation, ROS production, and mitochondrial damage in neutrophils isolated from dairy cows with SCH with or without silencing of ORAI1 or the use of a ROS scavenger.

\section{MATERIALS AND METHODS}

The Ethics Committee on the Use and Care of Animals at Heilongjiang Bayi Agricultural University (Daqing, China) approved the animal study protocol (no. 2020120701).

\section{Animals}

Lactating Holstein cows at $2 \mathrm{~d}$ postpartum from a commercial dairy farm (Heilongjiang Province, China) received a routine physical examination by the attending veterinarian to ensure absence of other co-morbidities: normal calf delivery, retained placenta, lameness, subclinical mastitis (measured by California Mastitis Test) and ketosis [with blood BHB $<1.2 \mathrm{mmol} / \mathrm{L}$ measured by a blood ketone meter (TNN-2, Yicheng Bioelectronics Technology Co. Ltd.)]. Subsequently, blood samples were collected before feeding and after milking. One set of samples $(5 \mathrm{~mL})$ were collected into sodium-heparin tubes, and after centrifugation at 1,400 $\times g$ for $10 \mathrm{~min}$ at room temperature, the plasma was harvested for measuring concentrations of calcium immediately using commercial kits (Changchun Huili Biotech Co. Ltd.) in a semi-automatic biochemical analyzer (RT-9600, Rayto). Another set of samples (20 mL) was collected into sodium citrate tubes for neutrophil isolation. A total of 10 cows with $\mathrm{Ca}^{2+}$ concentrations of 1.38 to 2.00 $\mathrm{mmol} / \mathrm{L}$ were deemed to have SCH and 10 cows with $>2.10 \mathrm{mmol} \mathrm{Ca}^{2+} / \mathrm{L}$ were deemed healthy (control). Characteristics of $\mathrm{SCH}$ and control cows are reported in Table 1. Milk yield was assessed with a portable semi-automatic milking equipment, and production at 0600 and $1500 \mathrm{~h}$ on sampling day measured with an electronic scale. Body condition score (scale of 1-5) was assessed by a experienced veterinarian using a 5-point system with 0.25 increments, with 1 being too thin and 5 being too obese (Ferguson et al., 1994).

Table 1. Characteristics of control and subclinical hypocalcemia (SCH) cows used in the present study

\begin{tabular}{lccccc}
\hline & \multicolumn{2}{c}{ Control $(\mathrm{n}=10)$} & & \multicolumn{2}{c}{$\mathrm{SCH}(\mathrm{n}=10)$} \\
\cline { 2 - 3 } \cline { 5 - 6 } Item & Median & Interquartile range & & \multirow{2}{*}{ Median } & Interquartile range \\
\hline Ca $(\mathrm{mmol} / \mathrm{L})$ & 2.34 & $2.29,2.41$ & & 1.85 & $1.80,1.92$ \\
Parity & 3 & 2,3 & 3 & 2,3 \\
DIM & 2 & 2,2 & 2 & 2,2 \\
Milk yield $(\mathrm{kg} / \mathrm{d})$ & 26.2 & $24.8,27.4$ & & 26.3 & $25.5,29.2$ \\
BW $(\mathrm{kg})$ & 609.5 & $606.6,615.3$ & & 611.7 & $601.0,618.4$ \\
BCS & 3.00 & $2.75,3.00$ & & 3.00 & $3.00,3.00$ \\
\hline
\end{tabular}




\section{Isolation and Culture of Neutrophils}

Blood samples $(18 \mathrm{~mL})$ were collected at $2 \mathrm{~d}$ postpartum before feeding via venipuncture in a $10-\mathrm{mL}$ centrifuge tube (9-mL blood sample per tube, Nunc) containing $1 \mathrm{~mL}$ of $3.82 \%$ sodium citrate (Solarbio). After mixing well, samples were transported to the laboratory at $4^{\circ} \mathrm{C}$ within $2 \mathrm{~h}$. A commercial Neutrophil Isolation Kit (Solarbio) was used according to the manufacturer's instructions. Briefly, blood was layered on separating solution followed by addition of PBS containing $0.02 \%$ EDTA and centrifugation at $800 \times g$ for $30 \mathrm{~min}$ at room temperature. Neutrophils were collected and centrifuged $300 \times g$ for $10 \mathrm{~min}$ at room temperature. The sediment was washed with PBS and erythrocytes lysed using red cell lysing reagent followed by centrifugation at $300 \times g$ for $10 \mathrm{~min}$ at room temperature. Neutrophils were washed twice with RPMI 1640 medium (Sigma) and resuspended in $5 \mathrm{~mL}$ of RPMI 1640 medium. After counting in a blood count plate (XB. K. 25. $0.10 \mathrm{~mm}$ $1 / 400 \mathrm{~mm}^{2}$, Shanghai Qiujing Biochemical Reagent Instrument Co. Ltd.), neutrophils were diluted to $2.0 \times$ $10^{6}$ cells/mL with RPMI 1640 medium for subsequent experiments described in the next section.

\section{Treatments}

Neutrophils from control $(\mathrm{n}=10)$ and SCH $(\mathrm{n}=10)$ cows were seeded in 6 -well plates at a density of $5 \times$ $10^{5}$ cells $/ \mathrm{mL}(2 \mathrm{~mL}$ per well, 3 replicates per cow for each treatment) or 24-well plates with round coverslips at a density of $5 \times 10^{5}$ cells $/ \mathrm{mL}(0.5 \mathrm{~mL}$ per well, 3 replicates per cow for each treatment) with RPMI 1640 medium at $37^{\circ} \mathrm{C}$ with $5 \% \mathrm{CO}_{2}$ for $1 \mathrm{~h}$. Cells in 6 -well plates and 24-well plates were treated with 50 $\mu M$ ORAI1 inhibitor 2-aminoethoxydiphenyl borate (2APB), $1 \mu M$ sarcoendoplasmatic $\mathrm{Ca}^{2+}$ ATPase inhibitor thapsigargin, $10 \mathrm{~m} M$ oxidative stress inhibitor $\mathrm{N}$-acetylcysteine (NAC), or dimethyl sulfoxide for 5 min, respectively, then stimulated with $100 \mathrm{n} M$ phorbol 12-myristate 13-acetate (PMA) for $3 \mathrm{~h}$. Cell treatments were divided into 8 groups: control, $\mathrm{SCH}$, $2 \mathrm{APB}+$ control, $2 \mathrm{APB}+\mathrm{SCH}$, thapsigargin + control, thapsigargin $+\mathrm{SCH}, \mathrm{NAC}+$ control, and $\mathrm{NAC}$ + SCH. Neutrophils from 6-well plates were used for ROS, NADPH analysis, and neutrophils from 24-well plates were used for NET formation, MPO and NE formation, calcium release, and mitochondrial imaging. Three replicates per cow were used for each assay.

\section{RNA Silencing Transfection}

For silencing studies, $2 \times 10^{6}$ cells/well (6-well plate) or $5 \times 10^{5}$ cells/well ( 24 -well plate with round coverslips) neutrophils from control cows $(\mathrm{n}=10)$ were seeded $1 \mathrm{~h}$ before the experiment and then transfected with $40 \mathrm{p} M$ ORAI1 small interfering (si)RNA (Shanghai GenePharma Co. Ltd.) or nontarget siRNA (siNEG; Shanghai GenePharma Co. Ltd.) using siRNA-Mate transfection reagent (Shanghai GenePharma Co., Ltd.) according to the manufacturer's protocol (Zhang et al., 2019), then stimulated with PMA for $3 \mathrm{~h}$. Neutrophils from 6-well plates were used for ROS, RT-PCR, and Western blotting, and cells from 24-well plates were used for NET formation and calcium release assays. Three technical replicates per cow were used for each assay. The ORAI1 siRNA sequence is 5'GCAACGUGCACAACCUCAATT3', 5'UUGAGGUUGUGCACGUUGCTT3'.

\section{Immunofluorescence for NET Formation Analysis}

To assess NET formation, neutrophils combined in round coverslips (from 24-well plates) from different groups were fixed with $4 \%$ paraformaldehyde for 15 min at $4^{\circ} \mathrm{C}$. After washing steps, the nuclei were stained with Hoechst 33342 dye (Beyotime) for 8 min at room temperature. Images were obtained with a confocal laser-scanning microscope (Leica TCS SP8; Leica) with $40 \times / 1.3$ numerical aperture differential interference contrast and analyzed with the instrument's software. The percentage of NET with all neutrophils visualized was calculated to assess NET formation.

To assess MPO and NE formation, neutrophils combined in round coverslips (from 24-well plates) from control and SCH cows were fixed with $4 \%$ paraformaldehyde for $30 \mathrm{~min}$ at room temperature and nonspecific binding sites were blocked with 3\% Albumin Bovine V (Biosharp), 5\% normal goat serum (Boster), and 0.5\% Triton (BioFROXX) in PBS (Biosharp) for $30 \mathrm{~min}$ at room temperature. Cells were then incubated with rabbit anti-MPO (1:500, Proteintech)-NE (1:500, Biorbyt) at $4^{\circ} \mathrm{C}$ in a humidified chamber overnight. Cells were rinsed 4 times with $\mathrm{PBS}$ and incubated with $\mathrm{Cy} 3$ goat anti-rabbit IgG (1:1,000, Beyotime) for $1 \mathrm{~h}$ at room temperature. After 4 washing steps, the nuclei were stained with Hoechst 33342 dye (Beyotime). Images were obtained with a confocal laser-scanning microscope (Leica TCS SP8; Leica).

\section{Transmission Electron Microscopy for Mitochondrial Imaging}

Neutrophils combined in round coverslips (from 24-well plates) from control and $\mathrm{SCH}$ cows were fixed overnight at $4{ }^{\circ} \mathrm{C}$ with $4 \%$ glutaraldehyde and washed with PBS, then fixed with $1 \%$ osmium tetroxide at room temperature under shaking in an Orbital shaker (TS-1000, Kylin Bell) for $1 \mathrm{~h}$. The fixed cells were 
dehydrated in different concentrations of ethanol (70, 80,90 , and $100 \%$ ) followed by addition of acetone and embedding agent to permeate cells. The permeated cells were polymerized and sectioned with an ultra-thin sectioning machine (Leica EM UC7) to stain with uranyl acetate and lead citrate. Sections were subsequently observed through transmission electron microscopy (JEM-2100Plus, JEOL Ltd.).

\section{Scanning Electron Microscopy for NET Formation Imaging}

Neutrophils combined in round coverslips (from 24well plates) from control and SCH cows were fixed with $2.5 \%$ glutaraldehyde overnight and dehydrated to glue to the plate and spray with gold as a conductor. Scanning electron microscopy was performed according to a method described previously (Yin et al., 2019) using a scanning electron microscope (SU-8010, Hitachi Ltd.) at a magnification of $8,000 \times$.

\section{Quantification of DNA for NET Production Analysis}

Neutrophil supernatant was collected and measured using Quant-iT PicoGreen dsDNA Assay Kit (Invitrogen) according to the manufacturer's protocol. Briefly, isolated neutrophils $\left(1 \times 10^{6}\right.$ cells/well $)$ were seeded into 96-well plates in $100 \mu \mathrm{L}$ of RPMI 1640 medium (phenol-red-free) and incubated with $10 \mathrm{mM}$ NAC, 1 $\mu M$ thapsigargin, or $50 \mu M 2 \mathrm{APB}$ for $5 \mathrm{~min}$, respectively, then stimulated with $100 \mathrm{n} M$ phorbol 12-myristate 13-acetate (PMA) for $3 \mathrm{~h}$. Then the aqueous working solution of the Quant-iT PicoGreen reagent was added to each well. Subsequently, fluorescence intensity was measured via a Fluorescence Spectrophotometer (SpectraMax Gemini EM, Molecular Devices) at $520 \mathrm{~nm}$ after excitation at $485 \mathrm{~nm}$. The level of extracellular DNA was quantified to assess the ability of neutrophils to produce NET.

\section{Calcium Release Assay (Mitochondrial Permeability Transition Pore)}

Neutrophils combined in round coverslips (from 24well plates) from control and $\mathrm{SCH}$ cows treated with thapsigargin and $2 \mathrm{APB}$, respectively, were used for calcein release measurement performed according to the Transition Pore Assay Kit following the manufacturer's instructions (I35103, Thermo Fisher Scientific). Cells were washed twice with modified Hanks' balanced salt solution buffer (14175095, Thermo Fisher Scientific), and then incubated for $15 \mathrm{~min}$ in sufficient amounts of labeling solution (consisting of $1 \mathrm{mM}$ calcein and $1 \mathrm{mM}$
$\mathrm{CoCl}_{2}$ ) and protected from light at $37^{\circ} \mathrm{C}$. After incubation, cells were washed with warm Hanks' balanced salt solution buffer to remove residual dye and minimize background. A confocal laser scanning microscope (Leica TCS SP8) was used to acquire fluorescence images.

\section{Measurement of ROS}

To measure intracellular ROS production, according to the manufacturer's instructions, neutrophils in 6-well plates treated with thapsigargin, 2APB, siORAI1, or siNEG were resuspended and $1 \times 10^{5}$ cells combined with $10 \mu M$ carboxy-2', $7^{\prime}$-dichlorodihydrofluorescein diacetate and incubated with the probe for $15 \mathrm{~min}$ at $37^{\circ} \mathrm{C}$. The voltage was determined based on untreated cells and the fluorescence measured at $488 \mathrm{~nm}$ (excitation) and $525 \mathrm{~nm}$ (emission) in a Beckman CytoFLEX FCM (Beckman Coulter). Approximately $1 \times 10^{4}$ cells were analyzed in each sample. Data were analyzed by FlowJo software (FlowJoX10.07; Becton, Dickinson and Company). Briefly, according to the y-axis defaults to side scatter and the $\mathrm{x}$-axis defaults to forward scatter, neutrophils were circled in the flow scatter plot, then ROS production was analyzed through calculating the percentage of fluorescence intensity (after subtracting the negative control) to total fluorescence intensity.

\section{Measurement of NADPH}

To measure NADPH, we collected cells in 6-well plates treated with thapsigargin and $2 \mathrm{APB}$, washed them twice with PBS ( $\mathrm{pH}$ 7.4) and centrifuged them for 5 min at $600 \times g$ at $4^{\circ} \mathrm{C}$. Cells were then treated with $0.2 \mathrm{~mL}$ of cold lysis solution for $2 \mathrm{~min}$ and centrifuged for 10 min at $12,000 \times g$ at $4^{\circ} \mathrm{C}$. The lysed cell suspension was incubated at $60^{\circ} \mathrm{C}$ for $30 \mathrm{~min}$ and $20 \mu \mathrm{L}$ was added to a 96-well plate. Subsequently, $90 \mu \mathrm{L}$ of alcohol dehydrogenase was added and incubated at $37^{\circ} \mathrm{C}$ for 10 min. Finally, $10 \mu \mathrm{L}$ of chromogenic solution was added to the plate and the mixture incubated at $37^{\circ} \mathrm{C}$ for 30 min. The absorbance values were measured at $450 \mathrm{~nm}$ and analyzed on a plate reader (Multiskan FC; Thermo Fisher Scientific).

\section{Cytosolic Calcium}

To measure cytosolic $\mathrm{Ca}^{2+}$ concentrations, isolated neutrophils from control cows transfected with siORAI1 or siNEG were washed with Tyrode buffer ( $\mathrm{pH}$ 7.4), stained with $3 \mu M$ Fluo-3AM (Biotinium) in the same buffer, and incubated at $37^{\circ} \mathrm{C}$ for $30 \mathrm{~min}$ (Zhang et al., 2019). Relative fluorescence was measured utilizing a Beckman CytoFLEX FCM (Beckman Coulter). 


\section{ORAl1 Quantification Via RT-PCR}

Total RNA of neutrophils from control cows transfected with siORAI1 or siNEG was extracted with TRIzol reagent (Invitrogen Corporation) according to the manufacturer's protocols. The cDNA was obtained using a PrimeScript RT reagent kit with gDNA Eraser (Takara Bio). The PCR reactions were performed in a Bio-Rad iCycler iQTM Real-Time PCR Detection System (Bio-Rad Laboratories Inc.). Primers used for bovine ORAI1 and ACTB are shown in Table 2. The mRNA abundance in each sample was normalized to $A C T B$, and quantified with the $2^{-\Delta \Delta \mathrm{CT}}$ method.

\section{Western Blotting for ORAl1 Protein Abundance}

Neutrophils from control cows transfected with siORAI1 or siNEG with RIPA buffer (Beyotime Biotechnology) containing protease inhibitors were lysed,
Table 2. Sequences of primers used for real-time PCR amplification

\begin{tabular}{ll}
\hline Gene & Primer (5' to 3$)$ \\
\hline Bovine ORAI1 & Forward: TTTGCCGTCCACTTCTAC \\
Bovine ACTB & $\begin{array}{l}\text { Reverse: CCTCTTTCCTCCACTTTCT } \\
\text { Forward: GCTAACAGTCCGCCTAGAAGCA } \\
\end{array}$ \\
\hline
\end{tabular}

incubated on ice for $30 \mathrm{~min}$, then centrifuged at 14,000 $\times g$ for 5 min at $4^{\circ} \mathrm{C}$. Total protein was collected and measured using the BCA protein assay kit (Beyotime). Subsequently, equal amounts of protein $(30 \mu \mathrm{g} /$ lane) were separated on $10 \%$ SDS-PAGE and electrotransferred onto a polyvinylidene difluoride membrane (Millipore Corp.). Then, the membrane was blocked for $1 \mathrm{~h}$ at room temperature in blocking buffer $(5 \%$ skim milk powder in Tris-buffered saline with Tween). The blocked membranes were incubated overnight at $4^{\circ} \mathrm{C}$ with specific antibodies for ORAI1 (1:1,000, Pro-
A

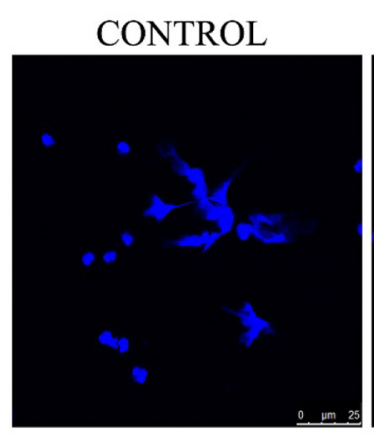

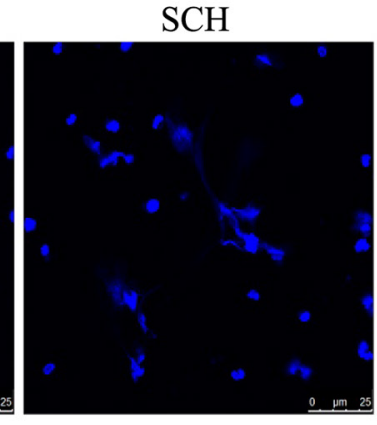

B

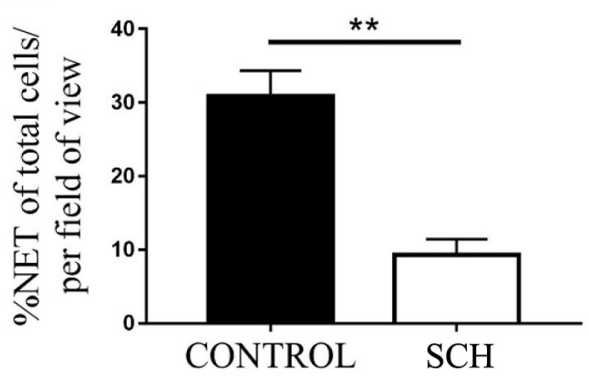

C

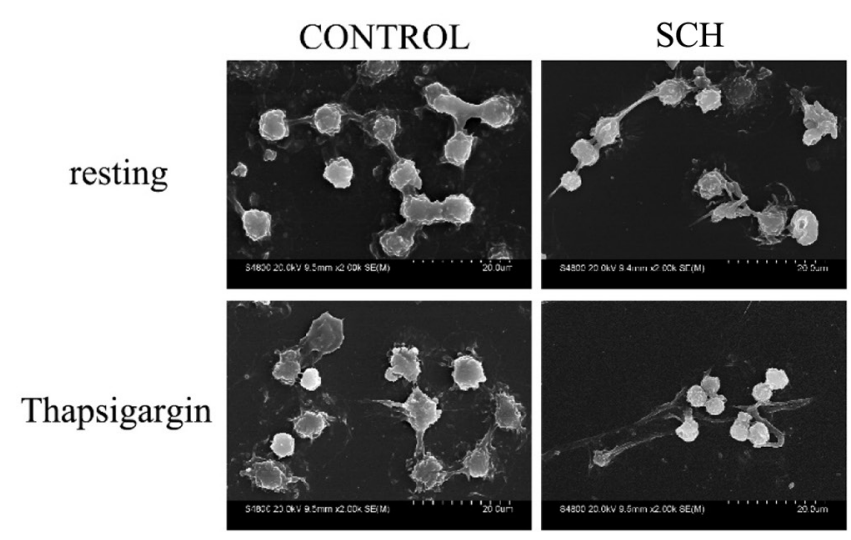

Figure 1. Visualization of neutrophil extracellular trap (NET) formation by neutrophils stimulated with phorbol 12-myristate 13-acetate (PMA) in control and subclinical hypocalcemia (SCH) dairy cows. (A) Visualization of NET formation using a fluorescence-based method in neutrophils from control (top panel) and SCH (bottom panel) after incubation with $100 \mathrm{n} M$ phorbol 12-myristate 13-acetate (PMA) for 3 h. Hoechst 33342 (Beyotime) was used to stain DNA. White bar indicates the scale $(25 \mu \mathrm{m})$; a representative photomicrograph is shown. (B) Percentage of NET formation was quantified as the percentage of neutrophils producing NET to total visualized neutrophils (mean \pm SEM, $\mathrm{n}$ $=10)$. ${ }^{* *} P<0.01$ denotes a significant difference from control ( $t$-test). (C) Representative scanning electron micrographs of NET producing neutrophils after co-cultivation with or without thapsigargin and PMA for $3 \mathrm{~h}$ (scale bar, $20 \mu \mathrm{m}$ ). 
teinTech) and $\beta$-actin (internal control; 1:1,000, Santa Cruz), respectively. Subsequently, the membranes were washed and incubated with horseradish peroxidaseconjugated secondary antibodies (3:5,000; Beyotime) for $30 \mathrm{~min}$ at room temperature. Protein abundance was visualized by enhanced chemiluminescence solution (Beyotime) using a protein imager (ProteinSimple). Band intensity was quantified using the Image Lab software (Amersham Imager 600, General Electric Company).

\section{Statistical Analysis}

Descriptive statistics were used to summarize calcium concentrations, parity, DIM, milk yield, BW, and BCS data of SCH and control cows. Neutrophil data are expressed as the means \pm standard error of means.
For each assay, triplicates were first averaged before statistics. Except for NET content with an intraassay \% coefficient of variation $<14.4$, intraassay $\%$ coefficient of variation for other assays was $<10$. The $\mathrm{n}$ represents the numbers of independent treatments. To compare groups, all data were first evaluated for normality with Shapiro-Wilk test and results indicated that they were normally distributed $(P>0.05)$. Subsequently, data of NET content and ROS from SCH and control cows, and data of NET content, DNA, ROS, cytosolic calcium, and mRNA and protein abundance of ORAI1 from siORAI1 and siNEG groups were analyzed using unpaired Student's $t$-tests; neutrophil NET content data, DNA, ROS, and NADPH from the thapsigargin-, 2APB-, and NAC-treated groups were analyzed by a one-way ANOVA followed by Duncan multiple comparisons test. The data were analyzed by SPSS Statistics (v.26;
A

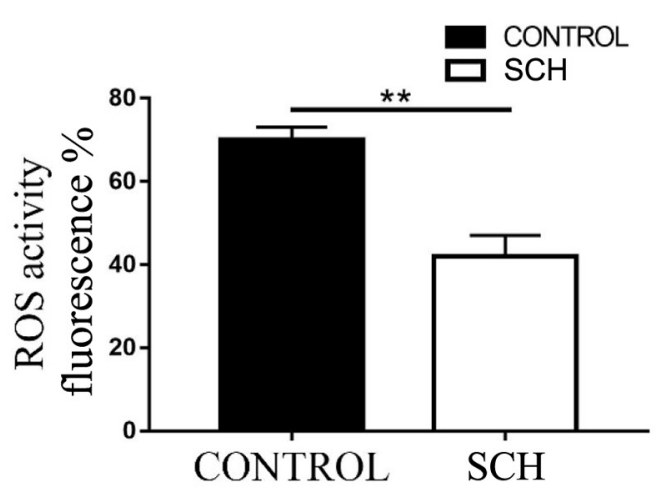

C

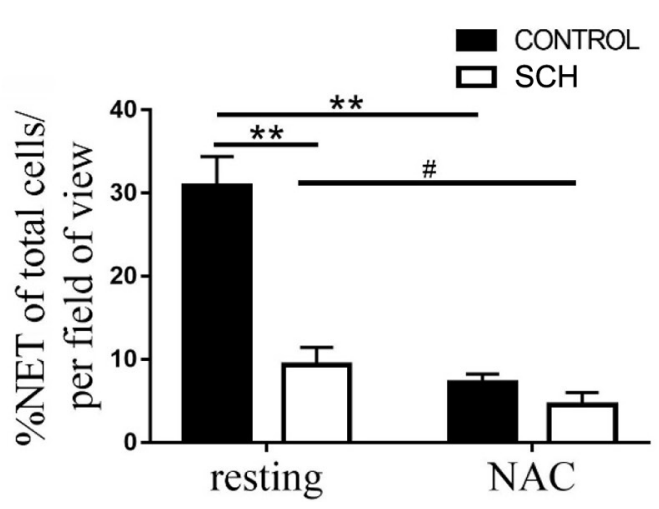

B

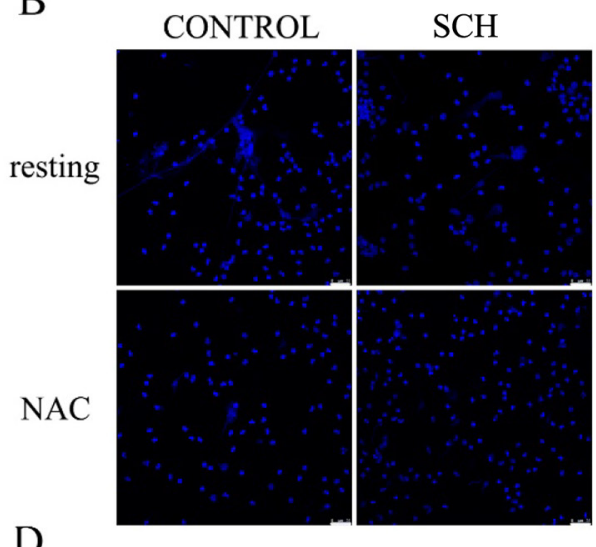

$\mathrm{D}$

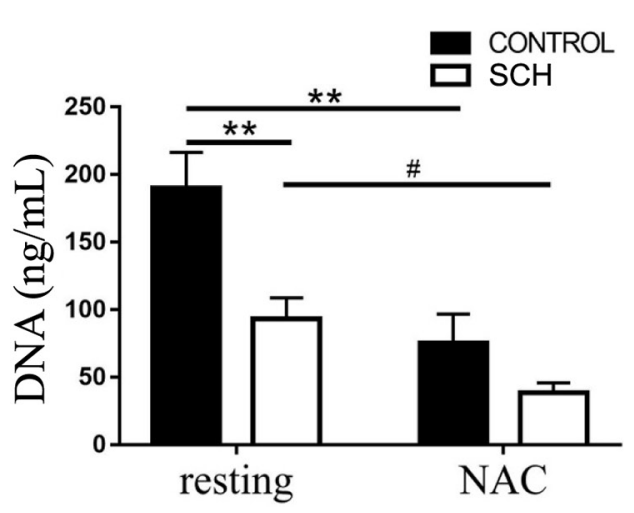

Figure 2. Effect of reactive oxygen species (ROS) production on neutrophil extracellular trap (NET) formation in neutrophils stimulated with phorbol 12-myristate 13-acetate (PMA) in control and subclinical hypocalcemia (SCH) dairy cows. (A) Reactive oxygen species production [means \pm SEM $(\mathrm{n}=10)]$ in neutrophils of control (left bars) and SCH cows (right bars). The ROS production was analyzed as the percentage of fluorescence intensity (after accounting for the negative control) to total fluorescence intensity. $* * P<0.01$ denotes a significant difference from control ( $t$-test). (B) Visualization of NET formation using a fluorescence-based method in neutrophils from control (left panel) and SCH cows (right panel) stimulated with PMA for $3 \mathrm{~h}$ in the presence or absence of $N$-acetylcysteine (NAC). The white bar indicates the scale (25 $\mu$ m). A representative photomicrograph is shown. (C) Percentage of NET formation in control (black bars) and SCH cows (white bars) was quantified as the percentage of neutrophils producing NET to the total visualized neutrophils. (D) DNA content [means \pm SEM ( $\mathrm{n}=10)$ ] in neutrophils from control (black bars) and SCH cows (white bars) stimulated with PMA for $3 \mathrm{~h}$ in the presence or absence of NAC. ${ }^{* *} P<0.01$ indicates significant difference from resting control. \#P<0.05 indicates significant difference from resting SCH (one-way ANOVA with a Duncan correction). 
IBM Corp.). A $P<0.05$ was considered statistically significant and $P<0.01$ highly significant.

\section{RESULTS}

\section{Neutrophil NET Formation in SCH and Control Cows}

As illustrated in Figure 1A-C, after PMA stimulation, immunofluorescence and scanning electron microscopy images revealed that control neutrophils exhibited irregular cell edges and released numerous filamentous structures, whereas in $\mathrm{SCH}$ the level of NET formation was reduced with far less typical DNA filaments and less diffused nuclei. In addition, in SCH the translocation of MPO to the nucleus was lower (Supplemental Figure 1A; https://figshare.com/ s/c8e710260b93c9d15304). Translocation of NE to the nucleus during NET formation was lower in SCH compared with control (Supplemental Figure 1B).

\section{Effect of Mitochondrial Dysfunction and ROS Production on NET Formation in SCH and Control Cows}

The spherical or rod-shaped nature of mitochondria was easily discernable (Supplemental Figure 2A; https://figshare.com/s/c8e710260b93c9d15304). In control, the crista of the mitochondria was clearly visible, whereas SCH was associated with broken crista, reduced mitochondrial permeability, and mitochondria were transformed into small vacuoles. As illustrated in Figure 2A and Supplemental Figure 2B, compared with control, SCH abrogated mitochondrial permeability transition pore (mPTP) opening, induced mitochondrial damage, and decreased production of ROS.

Compared with control, SCH neutrophils treated with NAC followed by a challenge with PMA had lower numbers of typical DNA filaments (Figure 2B). Furthermore, the DNA content was significantly lower in
A

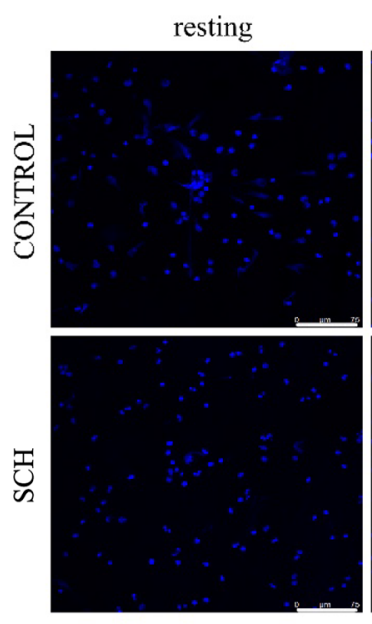

$\mathrm{C}$

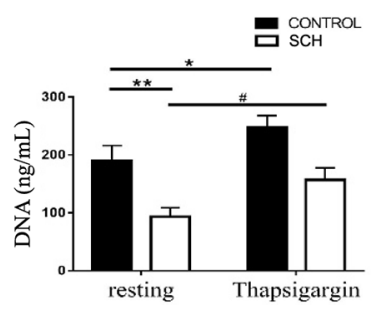

Thapsigargin

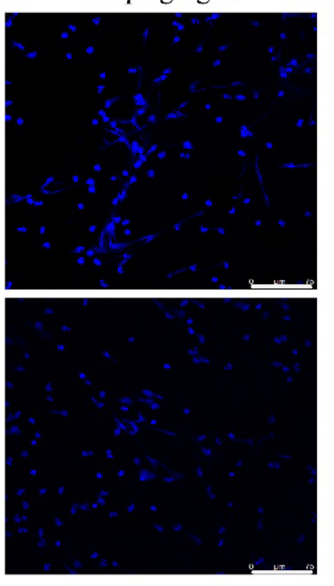

$\mathrm{D}$

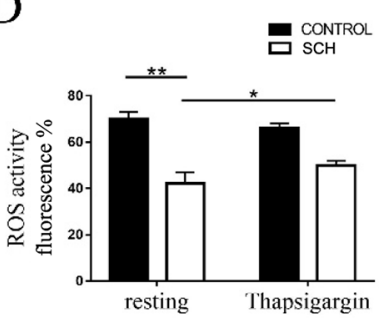

B

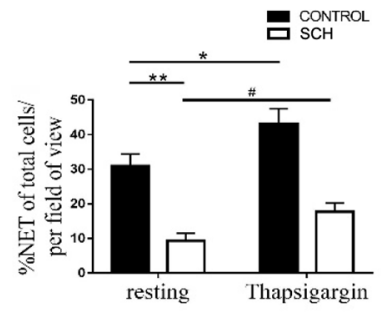

$\mathrm{E}$

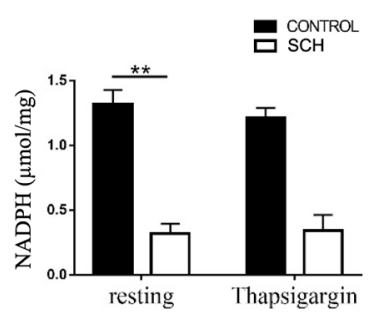

Figure 3. Effects of calcium on neutrophil extracellular trap (NET) formation of neutrophils stimulated with phorbol 12-myristate 13-acetate (PMA) in control and subclinical hypocalcemia (SCH) dairy cows. (A) Visualization of NET formation using a fluorescence-based method in neutrophils from control (left panel) and SCH cows (right panel) stimulated with PMA in the presence or absence of thapsigargin, respectively. Hoechst 33342 (Beyotime) was used to stain DNA. White bar indicates the scale $(75 \mu \mathrm{m})$. A representative photomicrograph is shown. (B) Percentage of NET formation in neutrophils of control and SCH cows was quantified as the percentage of neutrophils producing NET to the total visualized neutrophils. $(\mathrm{C})$ DNA content [means \pm SEM $(\mathrm{n}=10)]$ in neutrophils from control (black bars) and SCH cows (white bars) stimulated with PMA in the presence or absence of thapsigargin, respectively. (D) Reactive oxygen species production [means \pm SEM ( $=$ 10)] in neutrophils of control (black bars) and SCH cows (white bars). Reactive oxygen species production was analyzed as the percentage of fluorescence intensity (after accounting for the negative control) to total fluorescence intensity. (E) NADPH activity [means \pm SEM (n $=10)]$ in neutrophils of control (black bars) and SCH cows (white bars). ${ }^{*} P<0.05,{ }^{*} P<0.01$ indicate significant difference from resting control. \#P $<0.05$ indicates significant difference from resting SCH (one-way ANOVA with a Duncan correction). 
$\mathrm{SCH}$, and NAC decreased DNA content in both groups of dairy cows (Figure 2C and 2D).

\section{Effect of ORAl1 on NET Formation in Neutrophils}

As illustrated in Figure 3A and Figure 4A, thapsigargin induced NET formation and 2APB decreased NET formation in both control and SCH groups. Content of DNA, ROS production, and NADPH oxidase activation were greater in thapsigargin-treated cells following the challenge with PMA, but incubation with $2 \mathrm{APB}$ after the challenge with PMA decreased DNA content, ROS production, and NADPH oxidase activation in both control and $\mathrm{SCH}$ groups (Figure 3B-E and Figure 4B-E). Protein and mRNA abundance in siORAI1 cells was lower (Figure $5 \mathrm{~A}-\mathrm{C}$ ), and $\mathrm{Ca}^{2+}$ concentration in siORAI1 cells also decreased (Figure 5D). Both NET formation and DNA content were lower in the siORAI1 cells (Figure 6A-C). In addition, intracellular ROS production also decreased in the siORAI1 cells (Figure 6D).

\section{DISCUSSION}

In this study, we explored whether ORAI1 plays a role in regulating NET formation in dairy cows with subclinical hypocalcemia. Our data showed that neutrophils isolated from cows with subclinical hypocalcemia had a defective ROS- and ORAI1-dependent ability to induce NET formation. Furthermore, our results revealed a novel mechanism whereby ORAI1-regulated mitochondrial dysfunction could maintain NET formation in neutrophils.

As the first cells that migrate to inflammatory sites, neutrophils can kill pathogens and regulate innate and adaptive immunity through secreting various mediators (Gougerot-Pocidalo et al., 2006). The NET structure is characterized by decondensed chromatin and forms an extracellular reticular structure that can get rid of bacteria (Kirchgessner et al., 2016; Papayannopoulos, 2018). The NET functions mainly through the action of granular proteins such MPO, NE, and cathepsin G
A

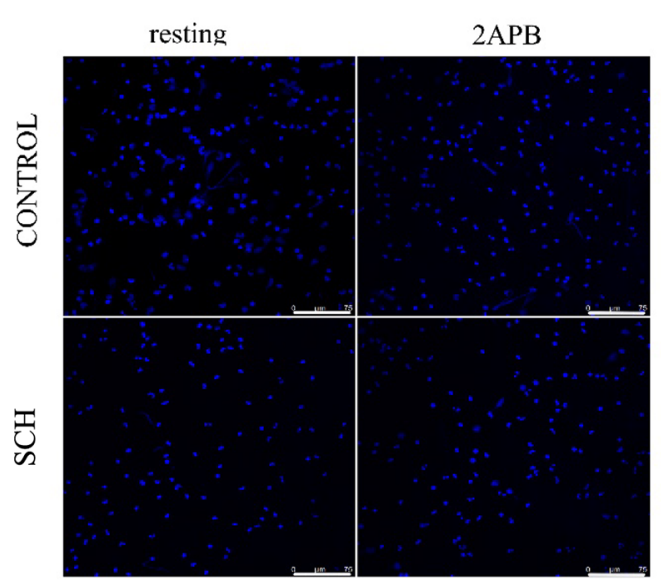

$\mathrm{C}$

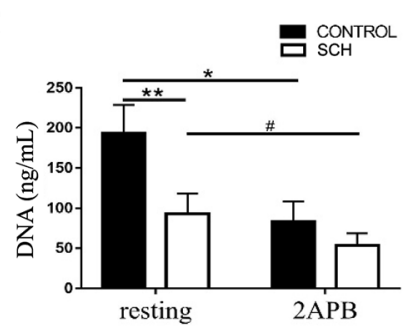

D

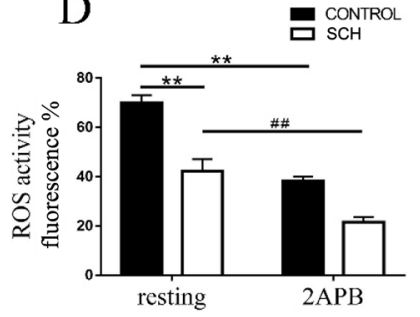

B

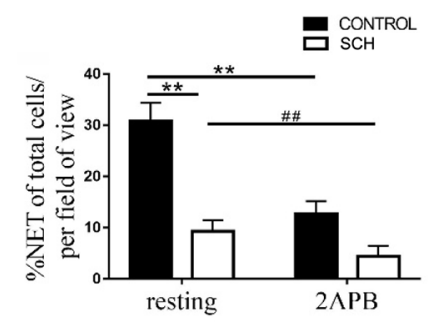

E

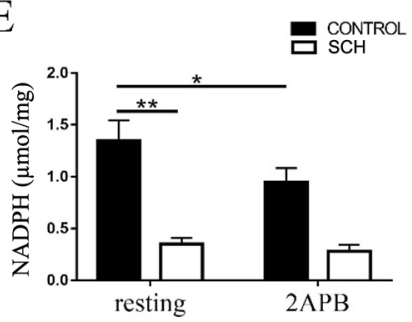

Figure 4. Effects of ORAI1 silencing on neutrophil extracellular trap (NET) formation of neutrophils stimulated with phorbol 12-myristate

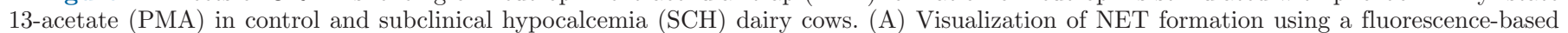

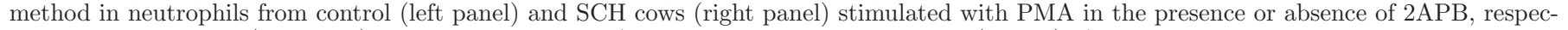

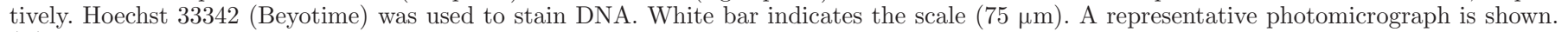

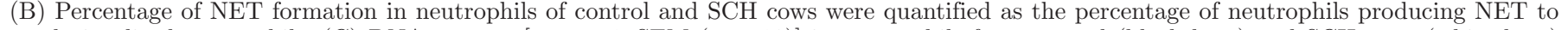

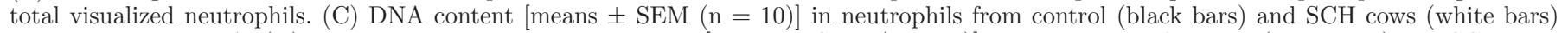

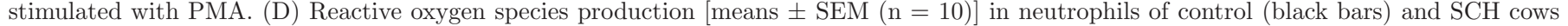

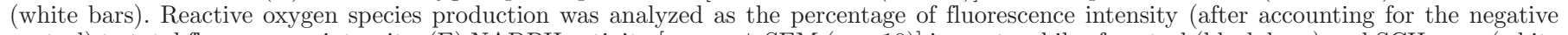

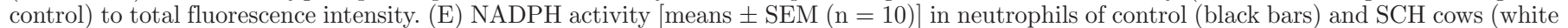

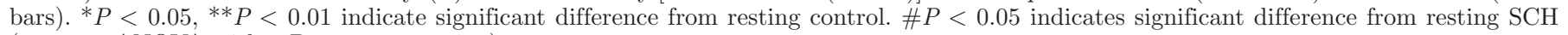
(one-way ANOVA with a Duncan correction). 
(Papayannopoulos et al., 2010). The MPO reaction generates hypochlorous acid and other highly reactive products to mediate efficient antimicrobial action (Klebanoff and Hamon, 1972; Harrison and Schultz, 1976). The MPO binds to chromatin and activates NE to translocate to the nucleus from azurophilic granules. The enzyme NE can modulate biological functions by promoting chemokine and cytokine activation and degradation, cytokine receptor shedding, proteolysis of cytokine binding proteins, and activation of different specific cell surface receptors (Lungarella et al., 2008; Papayannopoulos et al., 2010). The present study demonstrated that PMA stimulation of neutrophils from cows with hypocalcemia led to lower NET production and DNA content of neutrophils, hence suggesting that hypocalcemia decreases the ability of neutrophils to capture and kill bacteria.

Formation of NET is dependent on the production of ROS and enables neutrophils to perform their antibacterial function via activation of NADPH oxidase (Rohm et al., 2014; Ravindran et al., 2019). The present study uncovered that NET formation in dairy cows with hypocalcemia was associated with mitochondrial damage including changeable mitochondrial morphology and mPTP opening and increased ROS production. Thus, mitochondrial damage associated with hypocalcemia directly leads to a decrease in NET formation and negatively affects the antibacterial function of neutrophils. The present study revealed that $\mathrm{SCH}$ induced neutrophil mitochondrial dysfunction and changed mitochondrial membrane potential. The fact that the ROS scavenger NAC decreased NET formation after PMA stimulation indicated that NET formation requires ROS.

Intracellular $\mathrm{Ca}^{2+}$, an important secondary messenger, regulates differentiation, growth, activity, and metabolism of immune cells. The main switch to activate the immune system is the store-operated $\mathrm{Ca}^{2+}$ entry (Shi et al., 2013), which is composed of the calcium channel proteins ORAI and stromal interaction molecule in the ER. In the present study, the reduction in NET formation after PMA stimulation along with the reversal of that effect in response to thapsigargin when ORAI was inhibited or silenced indicated that
A

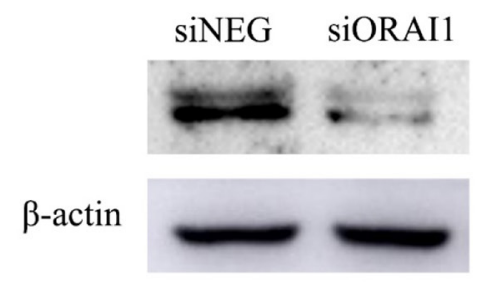

$\mathrm{C}$

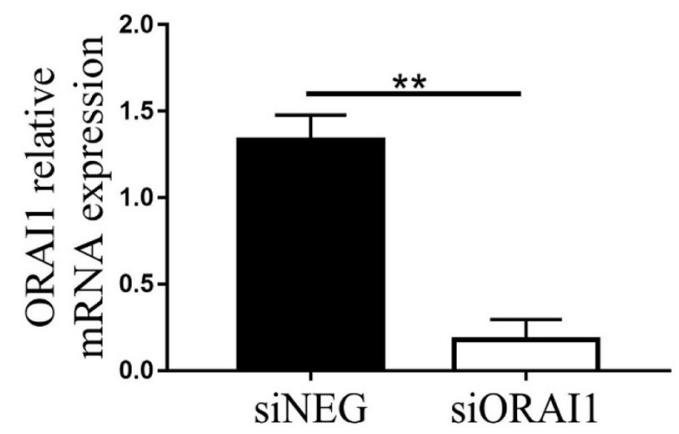

B

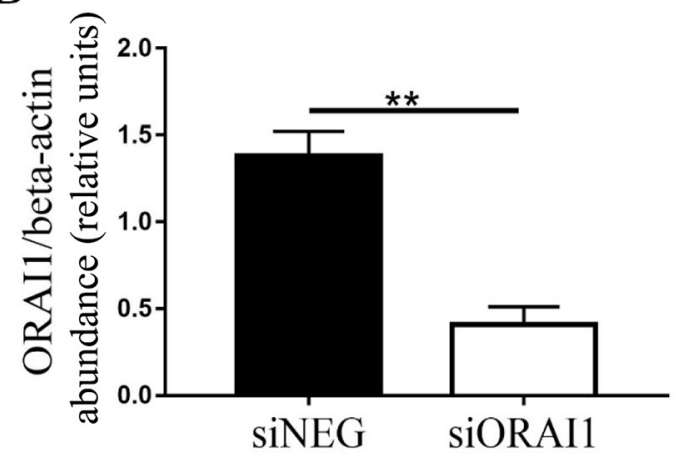

D

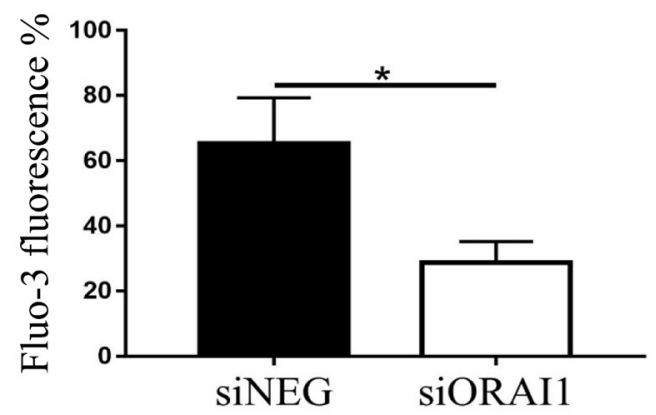

Figure 5. Effects of small interfering ORAI1 (siORAI1) on intracellular $\mathrm{Ca}^{2+}$ concentration in neutrophils from dairy cows. (A) Western blot analysis in control cells transfected with nontarget small interfering (si)RNA (siNEG; left) and siORAI1 (right) ORAI1. (B) Relative protein abundance in control cells transfected with siNEG (left) and siORAI1 (right) ORAI1. (C) Relative mRNA abundance of ORAI1. (D) Quantification of intracellular $\mathrm{Ca}^{2+}$ abundance was analyzed as the percentage of fluorescence intensity (after accounting for the negative control) to total fluorescence intensity [means \pm SEM $(\mathrm{n}=10)]$. ${ }^{*} P<0.05,{ }^{*} P<0.01$ indicates a significant difference from siNEG $(t$-test). 

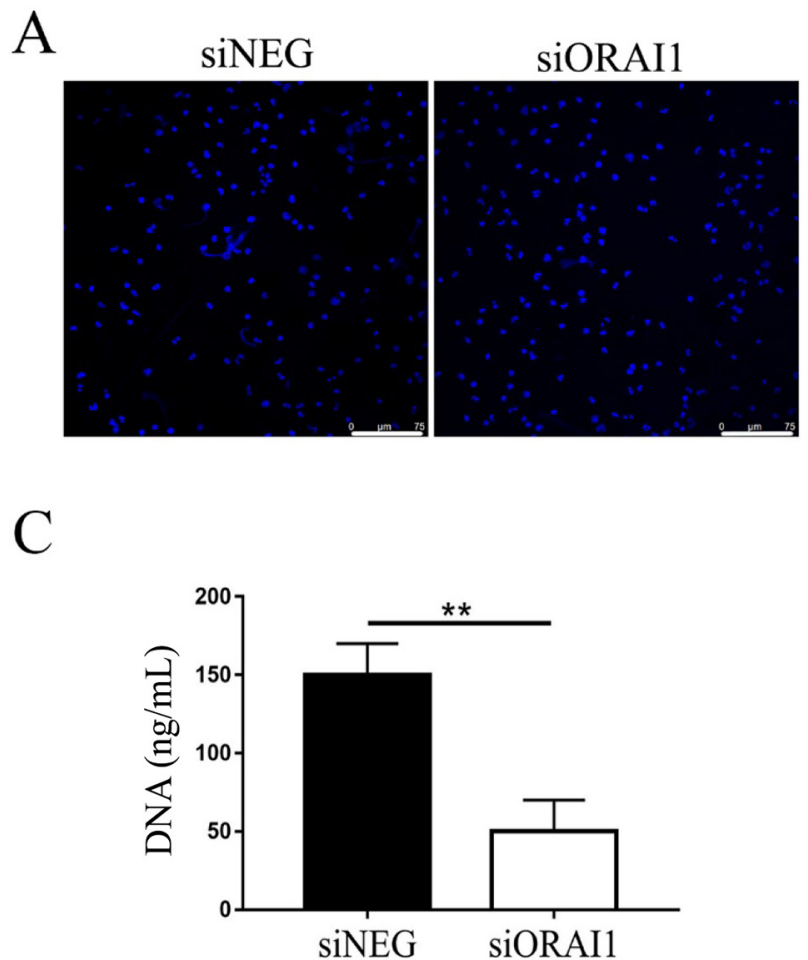

B

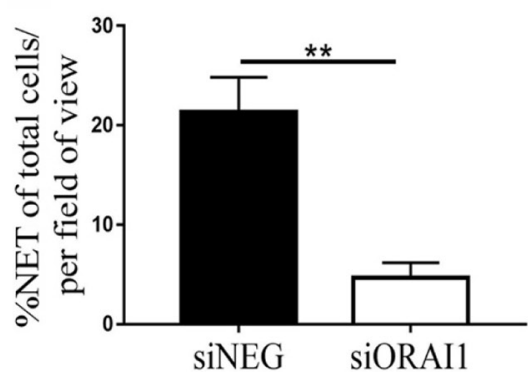

D

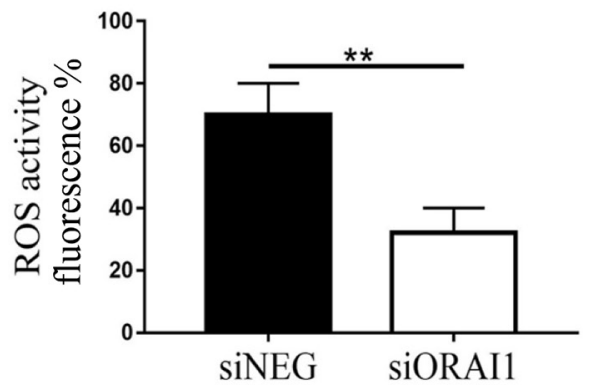

Figure 6. Effect of small interfering ORAI1 (siORAI1) on the production of neutrophil extracellular trap (NET) and reactive oxygen species in cow neutrophils. (A) Visualization of NET formation in neutrophils from control cells transfected with nontarget small interfering (si)RNA (siNEG; left) and siORAI1 (right) and stimulated with phorbol 12-myristate 13-acetate (PMA) using a fluorescence-based method. Hoechst 33342 (Beyotime) was used to stain DNA. The white bar indicates the scale $(75 \mu \mathrm{m})$. (B) The percentage of NET in neutrophils were quantified as the percentage of neutrophils producing NET to the total visualized neutrophils. (C) DNA content in neutrophils $[\mathrm{mean} \pm \mathrm{SEM}(\mathrm{n}=10)]$. (D) Reactive oxygen species production in neutrophils of siNEG (black bar) and siORAI1 (gray bar) [mean \pm SEM ( $\mathrm{n}=10$ )]. Reactive oxygen species production was analyzed as the percentage of fluorescence intensity (after accounting for the negative control) to total fluorescence intensity. ${ }^{* *} P<0.01$ indicates a significant difference from siNEG $(t$-test).

ORAI1 participates in NET formation in dairy cows. Intracellular $\mathrm{Ca}^{2+}$ was reported to increase ROS production in mitochondria (Gorlach et al., 2006). In the present study, thapsigargin increased intracellular $\mathrm{Ca}^{2+}$ concentration to regulate mitochondrial function including increased ROS production, enhanced mitochondrial membrane potential, and NAPDH content. The fact that inhibition or silencing of ORAI1 decreased intracellular $\mathrm{Ca}^{2+}$ concentrations and reduced ROS production and NAPDH content underscored the role of intracellular $\mathrm{Ca}^{2+}$ in mitochondrial function.

In vivo, availability of $\mathrm{Ca}^{2+}$ to maintain homeostasis is regulated by PTH, $1,25(\mathrm{OH})_{2} \mathrm{D}_{3}$, and $\mathrm{Ca}^{2+}$ itself (Honda et al., 2007; Nagai et al., 2011). Lower $1,25(\mathrm{OH})_{2} \mathrm{D}_{3}$ concentration (Vieira-Neto et al., 2017) and damaged PTH were considered to be a characteristic of dairy cows with hypocalcemia, and one of our earlier studies also confirmed greater $\mathrm{Ca}^{2+}$ concentrations in neutrophils and greater ORAI1 abundance in dairy cows with hypocalcemia (Zhang et al., 2019). Although the present data suggested that ORAI1-induced intracellular $\mathrm{Ca}^{2+}$ concentrations were sensitive to NET formation, the influence of the peripheral blood microenvironment in cows with hypocalcemia on NET formation remains to be determined.

In conclusion, $\mathrm{SCH}$ can reduce NET formation, the state of mPTP, ROS production, and NADPH oxidase activation in neutrophils in dairy cows. Decreased intracellular $\mathrm{Ca}^{2+}$, and especially ORAI1, regulated ROS production to cause mitochondrial damage and reduce NET formation in dairy cows with SCH. As such, the ability of neutrophils to respond to stimuli during the early postpartum period was severely curtailed, helping explain why dairy cows with hypocalcemia are more susceptible to infectious diseases.

\section{ACKNOWLEDGMENTS}

The study was supported by grants from the National Natural Science Foundation of China (Beijing; grant no. U20A2062) and Heilongjiang Province Natural Science Foundation Excellence Youth Project (Heilongjiang, China; grant no. YQ2020C035). The authors have not stated any conflicts of interest. 


\section{REFERENCES}

Brinkmann, V. 2018. Neutrophil extracellular traps in the second decade. J. Innate Immun. 10:414-421. https://doi.org/10.1159/ 000489829.

Chen, Q., Y. Zhou, L. Zhou, Z. Fu, C. Yang, L. Zhao, S. Li, Y. Chen, Y. Wu, Z. Ling, Y. Wang, J. Huang, and J. Li. 2020. TRPC6dependent $\mathrm{Ca}(2+)$ signaling mediates airway inflammation in response to oxidative stress via ERK pathway. Cell Death Dis. 11:170. https://doi.org/10.1038/s41419-020-2360-0.

Ferguson, J. D., D. T. Galligan, and N. Thomsen. 1994. Principal descriptors of body condition score in Holstein cows. J. Dairy Sci. 77:2695-2703. https://doi.org/10.3168/jds.S0022-0302(94)77212 $-\mathrm{X}$.

Goff, J. P. 2008. The monitoring, prevention, and treatment of milk fever and subclinical hypocalcemia in dairy cows. Vet. J. 176:50-57. https://doi.org/10.1016/j.tvjl.2007.12.020.

Gorlach, A., P. Klappa, and T. Kietzmann. 2006. The endoplasmic reticulum: Folding, calcium homeostasis, signaling, and redox control. Antioxid. Redox Signal. 8:1391-1418. https://doi.org/10 .1089 /ars.2006.8.1391.

Gougerot-Pocidalo, M. A., C. Elbim, P. M. Dang, and J. El Benna. 2006. Déficits fonctionnels primitifs des polynucléaires neutrophiles (Primary immune deficiencies in neutrophil functioning). Presse Med. 35:871-878. https://doi.org/10.1016/S0755-4982(06)74706 $-6$.

Harrison, J. E., and J. Schultz. 1976. Studies on the chlorinating activity of myeloperoxidase. J. Biol. Chem. 251:1371-1374. https://doi .org/10.1016/S0021-9258(17)33749-3.

Honda, H., N. Hosaka, and T. Akizawa. 2007. Kidney as a regulating organ for calcium and phosphate homeostasis. Clin. Calcium $17: 654-658$.

Immler, R., S. I. Simon, and M. Sperandio. 2018. Calcium signalling and related ion channels in neutrophil recruitment and function. Eur. J. Clin. Invest. 48(Suppl 2):e12964. https://doi.org/10.1111/ eci.12964.

Jawor, P. E., J. M. Huzzey, S. J. LeBlanc, and M. A. von Keyserlingk. 2012. Associations of subclinical hypocalcemia at calving with milk yield, and feeding, drinking, and standing behaviors around parturition in Holstein cows. J. Dairy Sci. 95:1240-1248. https:// doi.org/10.3168/jds.2011-4586.

Kirchgessner, T. G., P. Sleph, J. Ostrowski, J. Lupisella, C. S. Ryan, X. Liu, G. Fernando, D. Grimm, P. Shipkova, R. Zhang, R. Garcia, J. Zhu, A. He, H. Malone, R. Martin, K. Behnia, Z. Wang, Y. C. Barrett, R. J. Garmise, L. Yuan, J. Zhang, M. D. Gandhi, P. Wastall, T. Li, S. Du, L. Salvador, R. Mohan, G. H. Cantor, E. Kick, J. Lee, and R. J. Frost. 2016. Beneficial and adverse effects of an LXR agonist on human lipid and lipoprotein metabolism and circulating neutrophils. Cell Metab. 24:223-233. https://doi.org/ 10.1016/j.cmet.2016.07.016.

Klebanoff, S. J., and C. B. Hamon. 1972. Role of myeloperoxidasemediated antimicrobial systems in intact leukocytes. J. Reticuloendothel. Soc. 12:170-196.

Lood, C., L. P. Blanco, M. M. Purmalek, C. Carmona-Rivera, S. S. De Ravin, C. K. Smith, H. L. Malech, J. A. Ledbetter, K. B. Elkon, and M. J. Kaplan. 2016. Neutrophil extracellular traps enriched in oxidized mitochondrial DNA are interferogenic and contribute to lupus-like disease. Nat. Med. 22:146-153. https://doi.org/10.1038/ nm.4027.

Lungarella, G., E. Cavarra, M. Lucattelli, and P. A. Martorana. 2008. The dual role of neutrophil elastase in lung destruction and repair. Int. J. Biochem. Cell Biol. 40:1287-1296. https://doi.org/10.1016/ j.biocel.2007.12.008.

Martinez, N., L. D. Sinedino, R. S. Bisinotto, E. S. Ribeiro, G. C. Gomes, F. S. Lima, L. F. Greco, C. A. Risco, K. N. Galvao, D. Taylor-Rodriguez, J. P. Driver, W. W. Thatcher, and J. E. Santos. 2014. Effect of induced subclinical hypocalcemia on physiological responses and neutrophil function in dairy cows. J. Dairy Sci. 97:874-887. https://doi.org/10.3168/jds.2013-7408.
Nagai, S., M. Okazaki, H. Segawa, C. Bergwitz, T. Dean, J. T. Potts Jr., M. J. Mahon, T. J. Gardella, and H. Juppner. 2011. Acute down-regulation of sodium-dependent phosphate transporter NPT2a involves predominantly the cAMP/PKA pathway as revealed by signaling-selective parathyroid hormone analogs. J. Biol. Chem. 286:1618-1626. https://doi.org/10.1074/jbc.M110.198416.

Neves, R. C., B. M. Leno, K. D. Bach, and J. A. A. McArt. 2018. Epidemiology of subclinical hypocalcemia in early-lactation Holstein dairy cows: The temporal associations of plasma calcium concentration in the first 4 days in milk with disease and milk production. J. Dairy Sci. 101:9321-9331. https://doi.org/10.3168/ jds.2018-14587.

Papayannopoulos, V. 2018. Neutrophil extracellular traps in immunity and disease. Nat. Rev. Immunol. 18:134-147. https://doi.org/10 $.1038 /$ nri.2017.105.

Papayannopoulos, V., K. D. Metzler, A. Hakkim, and A. Zychlinsky. 2010. Neutrophil elastase and myeloperoxidase regulate the formation of neutrophil extracellular traps. J. Cell Biol. 191:677-691. https://doi.org/10.1083/jcb.201006052.

Ravindran, M., M. A. Khan, and N. Palaniyar. 2019. Neutrophil extracellular trap formation: Physiology, pathology, and pharmacology. Biomolecules 9:365. https://doi.org/10.3390/biom9080365.

Rohm, M., M. J. Grimm, A. C. D'Auria, N. G. Almyroudis, B. H. Segal, and C. F. Urban. 2014. NADPH oxidase promotes neutrophil extracellular trap formation in pulmonary aspergillosis. Infect. Immun. 82:1766-1777. https://doi.org/10.1128/IAI.00096-14.

Shi, X., Y. Bi, W. Yang, X. Guo, Y. Jiang, C. Wan, L. Li, Y. Bai, J. Guo, Y. Wang, X. Chen, B. Wu, H. Sun, W. Liu, J. Wang, and C. $\mathrm{Xu}$. 2013. Ca2+ regulates T-cell receptor activation by modulating the charge property of lipids. Nature 493:111-115. https://doi.org/ 10.1038 /nature11699.

Thilsing-Hansen, T., R. J. Jorgensen, and S. Ostergaard. 2002. Milk fever control principles: A review. Acta Vet. Scand. 43:1-19. https: //doi.org/10.1186/1751-0147-43-1.

Vieira Neto, R. J., K. C. S. Teixeira, M. M. Guerreiro, and M. A. Montenegro. 2017. Paranasal sinus disease in children with headache. J. Child Neurol. 32:1014-1017. https://doi.org/10.1177/ 0883073817724696.

Wang, Y., Y. Wang, J. Wu, C. Liu, Y. Zhou, L. Mi, Y. Zhang, and W. Wang. 2019. PRAK is required for the formation of neutrophil extracellular traps. Front. Immunol. 10:1252. https://doi.org/10 $.3389 /$ fimmu.2019.01252.

Wei, Z., J. Wang, Y. Wang, C. Wang, X. Liu, Z. Han, Y. Fu, and Z. Yang. 2019. Effects of neutrophil extracellular traps on bovine mammary epithelial cells in vitro. Front. Immunol. 10:1003. https: //doi.org/10.3389/fimmu.2019.01003.

Yin, K., Z. Yang, Y. Gong, D. Wang, and H. Lin. 2019. The antagonistic effect of $\mathrm{Se}$ on the $\mathrm{Pb}$-weakening formation of neutrophil extracellular traps in chicken neutrophils. Ecotoxicol. Environ. Saf. 173:225-234. https://doi.org/10.1016/j.ecoenv.2019.02.033.

Zhang, B., H. Guo, W. Yang, M. Li, Y. Zou, J. J. Loor, C. Xia, and C. Xu. 2019. Effects of ORAI calcium release-activated calcium modulator 1 (ORAI1) on neutrophil activity in dairy cows with subclinical hypocalcemia. J. Anim. Sci. 97:3326-3336. https://doi .org/10.1093/jas/skz209.

\section{ORCIDS}

Bingbing Zhang ๑ https://orcid.org/0000-0002-1869-9652

Xinru Ma @ https://orcid.org/0000-0003-2392-8015

Juan J. Loor (®) https://orcid.org/0000-0003-1586-4365

Qianming Jiang () https://orcid.org/0000-0001-9522-4856

Han Guo @ https://orcid.org/0000-0002-6386-4100

Chuang Xu ৫ https://orcid.org/0000-0002-0377-1439

Wei Yang ( https://orcid.org/0000-0003-3440-2190 